\title{
A model of stimulus induced epileptic spike-wave discharges
}

\author{
Peter N. Taylor, Gerold Baier, Sydney S. Cash, Justin Dauwels, Jean-Jacques Slotine, Yujiang Wang
}

\begin{abstract}
Recent clinical and experimental evidence suggests that the spike-wave discharges (SWD) of absence seizures result from local activity within a hyperexcitable cortical region with rapid generalization through thalamocortical networks. The cortical focus is said to react more strongly to stimulation than other areas. We seek to develop a model which is in agreement with these recent experimental findings and suggest a possible explanation.

In this study we extend an existing neural field model of thalamocortical interaction to account for multiple cortical regions which are connected according connectivity inferred from a clinically diagnosed epileptic patient. We stimulate at different model electrodes and investigate the resulting seizure duration.

We observe that stimulation of only a small subset (11\%) of model electrodes can lead to the rapid generalisation of SWD via both corticocortical and thalamocortical pathways. We find that the resulting model dynamics (seizure duration) varies significantly dependent upon the nodes stimulated and the amplitude of the stimulus.

Our model indicates that heterogeneities in corticocortical connectivity could serve as a possible reason for the cortical focus and provides a platform for in silico hypothesis generation in complement to in vivo hypothesis validation.
\end{abstract}

Index Terms-Epilepsy, EEG, Mathematical modelling, Spatiotemporal patterns, Spike-wave

\section{INTRODUCTION}

$\mathbf{E}$ PILEPSY is a neurological disorder characterised by abnormal brain rhythms recorded at the macroscopic level of the EEG electrode. In many patients with absence epilepsy this abnormal rhythm is manifested as a rapid spike followed by a slow wave which is highly synchronised across multiple brain regions and has a hallmark frequency of around $3 \mathrm{~Hz}$ [1]. Crucially, despite the high degree of synchrony, spatial differentiation can be made between recorded electrodes and between the recordings of different patients [2], [3], [4].

While generalized epilepsy is traditionally thought to involve widespread cortical areas recent evidence suggests there may be greater focality. For example, Luttjohann et al showed a heightened response to stimulation of the somatosensory cortex as opposed to stimulation of the motor cortex [5]. This variation in response was shown to be more pronounced in

P.N. Taylor \& J. Dauwels are with the School of Electrical \& Electronic Engineering Nanyang Technological University. Singapore. ptaylor@ntu.edu.sg

Y. Wang is with the Manchester Interdisciplinary Biocentre, University of Manchester, 131 Princess St., Manchester, M1 7DN, UK

G. Baier is with Modelling of Biological Processes, Centre for Organismal Studies, BioQuant BQ18, University of Heidelberg, Germany

S.S. Cash is with Massachusetts General Hospital and Harvard Medical School, Cambridge, MA, USA

J.J. Slotine is with Department of Mechanical Engineering and Department of Brain and Cognitive Sciences, Massachusetts Institute of Technology, Cambridge, MA, USA epileptic WAG/Rij rats than in healthy Wistar rats. Furthermore, Zheng et al showed that in order to induce a seizure in the GAERS strain of epileptic rats a stimulation of lower amplitude was required in some cortical regions than others [6]. Significant evidence suggests the thalamus also plays a key role in epileptic absence seizures (see e.g. [7] and references therein). This mounting evidence is in support of the 'cortical focus theory' [8], [9] and has the implication that a specific cortical region is responsible for seizure genesis which is the propagated and maintained through thalamocortical circuits. Currently the underlying reason for the suggested somatosensory cortical focus in WAG/Rij rats is not fully known.

Using fMRI, the somatosensory cortex was shown to have increased activity during seizures in the rat [10]. Recent fMRI studies of humans have revealed other focal regions have increased activity during seizure. These individual patterns of increased activity are consistent between seizures within a patient but different between patients [11] and often multifocal. However, despite this variation all but one patient had increased activation in the thalamus, further motivating thalamic involvement in seizures.

Experimental observations by Steriade et al [12], amongst others, led Destexhe [13] to develop a mathematical model of SWD which contains thalamocortical interactions at the scale of individual neurons. At the macroscopic scale of populations of neurons, Robinson et al developed a model of thalamocortical interaction capable of generating SWD which neglected spatial variation [14], [15], [16]. The model of Goodfellow et al [17] showed, using homogeneous connectivity, intermittent transitions to SWD. The neural field models of Wilson \& Cowan [18] and Amari [19] have been extended to demonstrate SWD [20], [21]. The model of Taylor \& Baier [20] was then further developed to account for heterogeneous connectivity based on human connectivity obtained through diffusion tensor imaging [22]. It did not however include explicit thalamic connectivity inferred from experimental observations. None of the aforementioned models have been shown to produce the significant spatial variation between channels in terms of the spike-wave waveform morphology at the whole brain scale as motivated in e.g. [4]. Perhaps the most detailed attempt at matching the morphology of the clinical EEG waveforms was by Holgado et al [23], however, this did not investigate any spatial variation.

Producing realistic epileptiform activity in both space and time at the large scale represents a significant challenge which we aim to investigate in this work. In this study we seek to bring together the the cortical focus theory and the testing 
of stimulation protocols in silico. Thus, we investigate the specific hypothesis that variations in response to stimulation is dependent on heterogeneous cortico-cortical connectivity.

\section{Methods}

\section{A. Model}

We use a two layer neural field model [19] containing one excitatory and one inhibitory population representative of pyramidal (PY) and inhibitory (IN) neural populations which are interconnected according to the connectivity parameters $C_{1 \ldots 9}$. At the local level, PY populations incorporate self excitation and project to the local IN population with strength $C_{2}$, reciprocal local connections from IN to PY are also included. Long range connections are both excitatory and inhibitory and are connected according to an adjacency matrix $A$ which is inferred from patient data (see section II.B for details). Additionally, thalamocortical connections are included through excitation of all PY populations from the TC variable, which also activates the RE population. Excitatory cortical populations excite both thalamic populations. In addition, the TC population excites the RE population and reciprocates by inhibition of TC and itself. In the situation that the size of $A=1$ the model reverts to a space independent model. This follows the connection scheme and model equations as presented in [24], which follows the the experimental evidence of these connections shown by Pinault \& O'Brien [7] and the references therein. This results in the following model equations which, when including an exponentially distributed noise process $W$ centred around zero and scaled by $\alpha$, take the form of the SDE:

$$
\begin{aligned}
d P Y(t)_{i}= & \tau_{1}\left(h_{p y}-P Y_{i}\right. \\
& +\sum_{j} C_{1} A_{j, i} f\left[P Y_{j}\right] \\
& -\sum_{j} C_{3} A_{j, i} f\left[I N_{j}\right] \\
& \left.+C_{9} f[T C]\right) d t \\
d I N(t)_{i}= & \tau_{2}\left(h_{i n}-I N_{i}+C_{2} f\left[P Y_{i}\right]\right) d t \\
d T C(t)= & \tau_{3}\left(h_{t c}-T C-C_{6} f[R E]\right. \\
& \left.+C_{7} f[P Y]\right) d t+\alpha d W \\
d R E(t)= & \tau_{4}\left(h_{r e}-R E-C_{4} f[R E]\right. \\
& \left.+C_{5} f[T C]+C_{8} f[P Y]\right) d t
\end{aligned}
$$

where $f[u]$ is the sigmoid function :

$$
f[u]=\left(1 /\left(1+\epsilon^{-u}\right)\right)
$$

and $u=P Y, I N, T C, R E$ with $\epsilon$ determining the steepness of the sigmoid. This follows the connection schematic as shown in figure 1. Since we are specifically interested in the impact of using a heterogeneous network, the time scale constants $\left(\tau_{1 \ldots 4}\right)$ and offset parameters $\left(h_{p y, i n, t c, r e}\right)$ are included homogeneously as in previous works [20].

Model solutions were computed numerically using the Euler-Maruyama solver and all results processed in MATLAB. For deterministic simulations (e.g. Figure 2a) the noise level

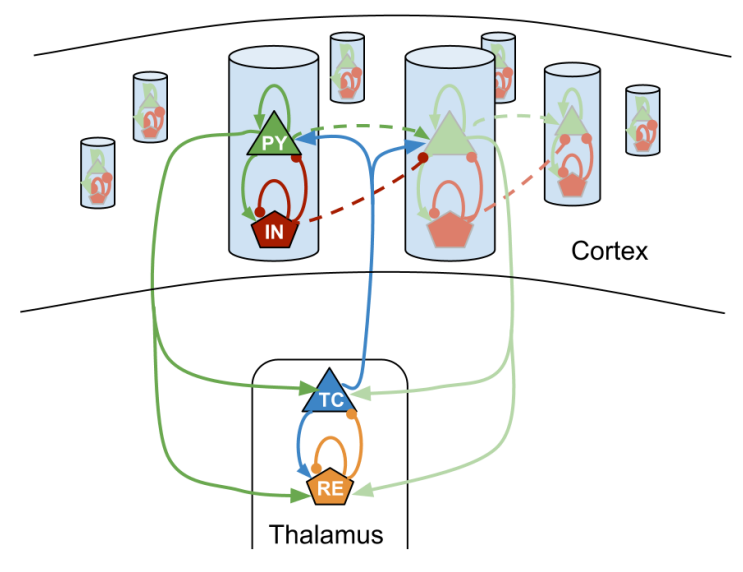

Fig. 1. Connectivity scheme of the model.

was set to zero. To investigate the impact of a stimulation at $t_{s}$ of strength $S$ the value in the noise vector was set to $S$ at this timepoint.

In the current formulation zero lag conduction delays are assumed as experimental evidence suggests the conduction velocity of axons can vary by more than an order of magnitude [25]. The investigation of subtle time variations between the proposed cortical focus and other areas, as shown by [8], is therefore left for future work as this would crucially depend on such information.

\section{B. Patient Data}

EEG data from a patient with clinically diagnosed epilepsy was used which lasted for a duration of 20 minutes. The data was sampled at $256 \mathrm{~Hz}$, referenced using a Laplacian montage, bandpass filtered between $0.5 \mathrm{~Hz}$ and $75 \mathrm{~Hz}$, then normalised (mean subtracted). Recordings used the standard 10-20 international 19 channel electrode placement system. This gives an EEG matrix of dimensions [time $\times$ number of channels (19)].

To infer connectivity between the electrode channels seizure epochs were removed and Pearson's cross correlation was computed. This results in a correlation matrix $M$ the diagonals equal to one of dimension $[19 \times 19]$. Correlations with a strength of less than $\lambda$ are set to zero. Since we wish to infer stronger self to self connectivity to represent local interaction we scale this through the parameter $w$.

$$
M_{i j}= \begin{cases}-M_{i j} & \text { if } M_{i j}<0 \\ 0 & \text { if } M_{i j}<\lambda \\ w & \text { if } i=j .\end{cases}
$$

The entire matrix is then scaled by the mean of the incoming connections:

$$
A=\frac{1}{\sum_{j} M_{i j}} M_{i j}
$$

In this study we wish to investigate the impact of a heterogeneous connection scheme on the resulting model dynamics. We therefore use the linear measure of cross correlation 
to infer our structural connectivity network. Ultimately the structural connectivity from the patient could be used e.g. inferred from diffusion tensor imaging (DTI). However, as a first approximation we use a functional connectivity network to serve our needs.

\section{RESULTS}

\section{A. Space independent model dynamics}

We begin through investigation of a space independent model with only one cortical compartment (i.e. $A=w$ ). Figure 2a shows a timeseries of the $P Y$ variable in the deterministic case, where the noise amplitude equals zero for all values except at $t \approx 9$ where a stimulus is applied to the TC variable. A transient response of multiple SWD oscillations can be seen in the model due to a deviation in phase space away from the fixed point, an excitable response. In this situation the excitable response is observed with the model located in the vicinity of a subcritical Hopf bifurcation. This bifurcation structure has also been used in other models of SWD [16]. The deviation ultimately returns to the stable fixed point at $t \approx 15$.

In the deterministic case a stimulus is required to elicit such a transient. Stochastic simulations incorporate a timevarying stimulus through the addition of a noise term. Figure $2 \mathrm{~b}$ shows a time series of such a simulation. Spontaneous seizures begin when the noise amplitude is large enough to elicit the transient response which ultimately terminates through a return to the stable fixed point. The background state in our model is therefore noisy oscillations around the stable focus which are of a low amplitude. In contrast, our seizure state can be considered as a transient response to a suprathreshold stimulus. This transient response takes the form of a high amplitude oscillation reminiscent of the morphology of an epileptic spike and wave discharge (cf. upper panels of figure $2 \mathrm{~b}$ and $2 \mathrm{c}$ ). Furthermore, the model reproduces similar activity to that of clinical recordings in the time-frequency domain. The lower panels of figures $2 b$ and $2 c$ show, using a Morlet wavelet transform, the time-frequency dependence during a simulated and clinical seizure. Both show dominance at $\approx 3 \mathrm{~Hz}$ with further harmonics at higher frequencies during the spike.

\section{B. Spatially extended model dynamics}

Clinical recordings of multiple scalp electrodes show a high degree of synchrony during the seizure [26] despite the known heterogeneity of cortico-cortical connections [27].

The incorporation of heterogeneities in the connectivity leads to some variation between channels during the simulated seizure, although high synchrony is present as described in [26]. Figure 3a (middle panel) shows an example timeseries of the stochastic model output with snapshots shown in the three right hand panels of the figure. Occasional seizures can be seen which appear spontaneous and are due to the noise input to the model. This compares with the clinical recording shown in figure $3 \mathrm{~b}$. Variation can be seen in terms of the amplitude of the SWD, the number of spikes per wave and the amplitude of the spike relative to the wave. Despite this

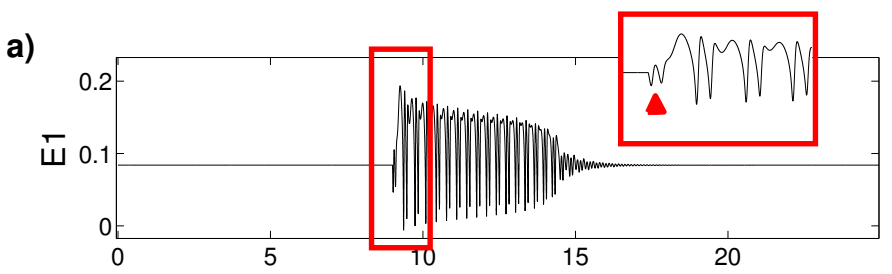

b)
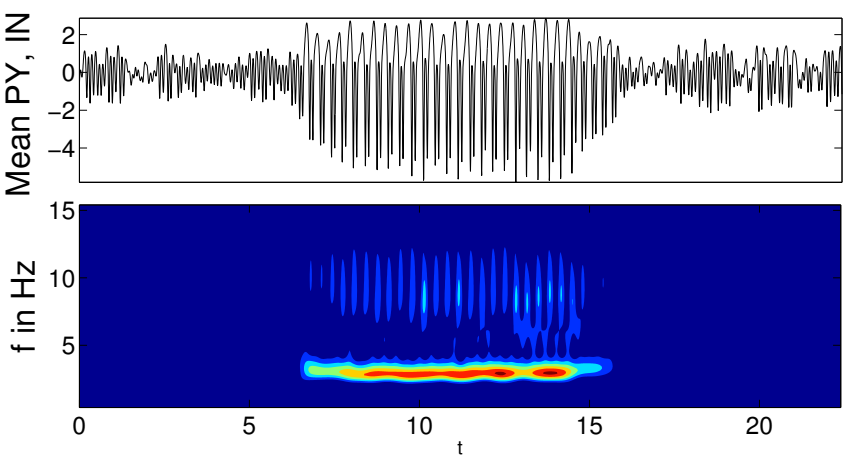

c)
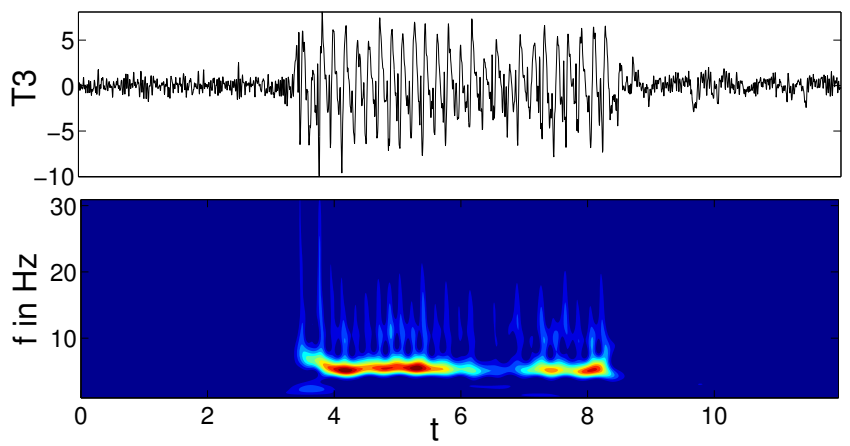

Fig. 2. a) Timeseries of PY variable during a simulation where a stimulus is applied to the TC variable at $t \approx 9$, indicated by the red arrow. b) Upper panel: timeseries of the cortical output (mean PY, IN) containing a seizure in the stochastic system. Lower panel: Time-frequency analysis using a Morlet avelet transform. c) Upper panel: timeseries of a clinical recording of an epileptic seizure from electrode T3. Lower panel: Time-frequency analysis using a Morlet avelet transform.

spatial variation there is a high degree of synchrony in both simulated and clinical EEG.

The left hand panels of figure 3 show a frequency spectrum for the ictal epochs (identified manually by visual inspection) in both simulated and clinical data. Again, all channels show dominance at around $3 \mathrm{~Hz}$ with some harmonics at higher frequencies.

\section{Stimulation}

One of the key benefits to computational modelling is the ability to probe the model and test hypotheses, specifically regarding stimulation.

Recent experimental findings in epileptic rats [5], [6], found differential response upon stimulation dependent upon the cortical location to be stimulated. We investigate the effect of a stimulus, in terms of the duration of the resulting seizure, to one and two cortical nodes in our deterministic model.

Figure $4 \mathrm{a}$ shows the a colour map indicative of seizure duration following stimulation to the corresponding cortical PY variables using a stimulus strength of -3 in the deterministic 
a)

운.

产

舟

मे

$0 \%$

o N

Q N W

a

¿ M

o M N M

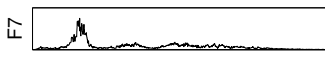

$\infty$

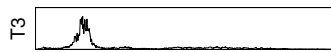

$+\sqrt{\square}$

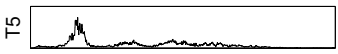

$\odot$ M

พ $M$

O N

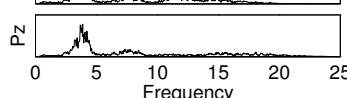

b)

눈

ปับ

悉

\&

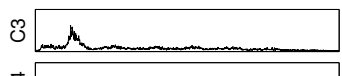

I0

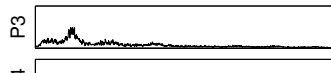

व

o

O

$\hat{L}$ _

$\infty$

$\stackrel{m}{r+2}$

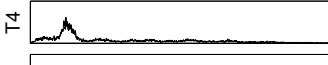

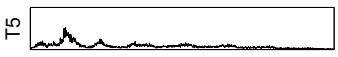

$\bullet$

L

0

\begin{tabular}{|lllllll}
\hline & & & & & & \\
\hline 0 & 5 & 10 & 15 & 20 & 25
\end{tabular}
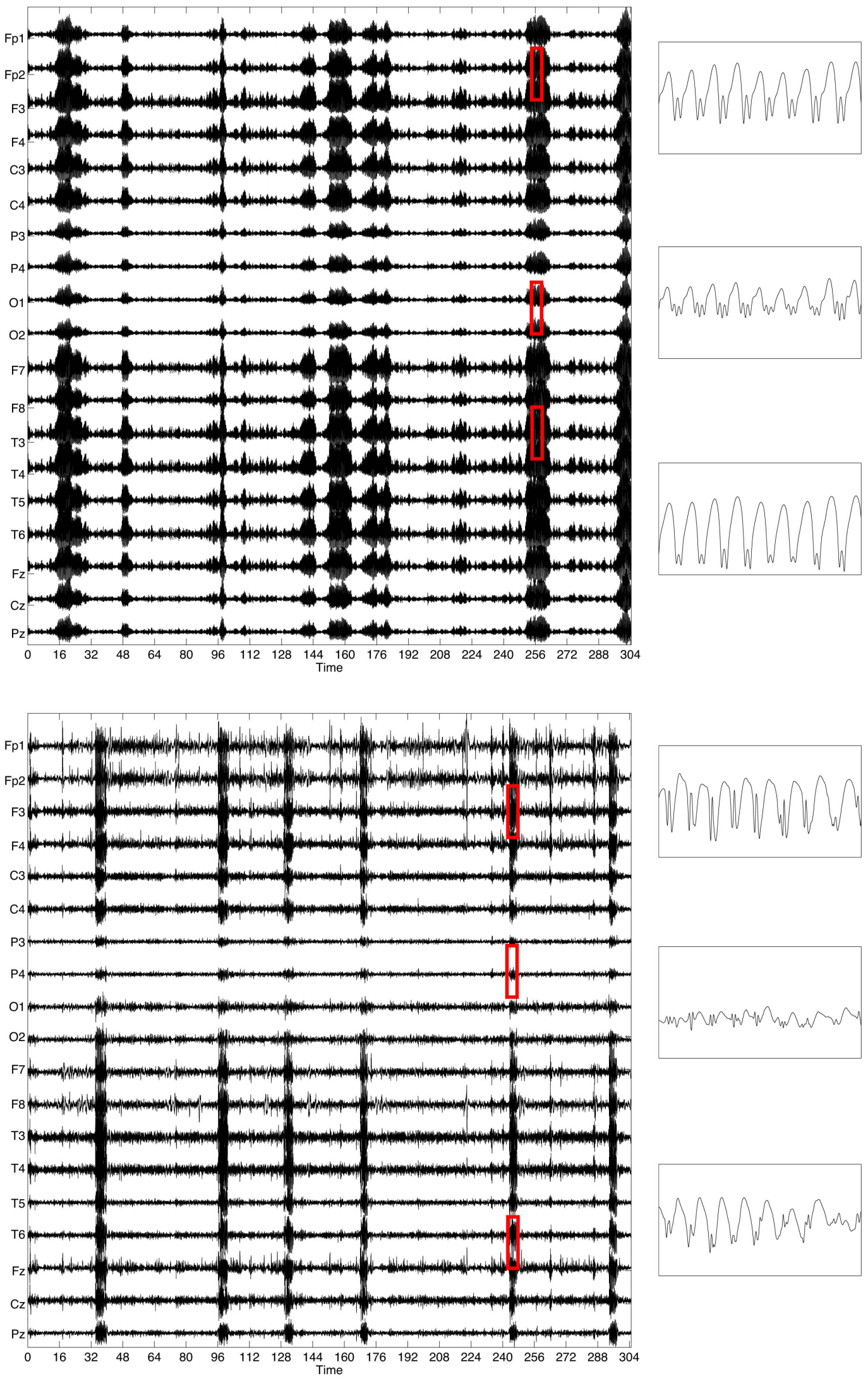

Fig. 3. Left panels: frequency spectrum of each channel. Centre panels: Timeseries of channels. Right panels: Close up of selected data indicated by the red boxes in the centre panel. a) Model output (mean of cortical variables). b) Clinical EEG recording. 
a)
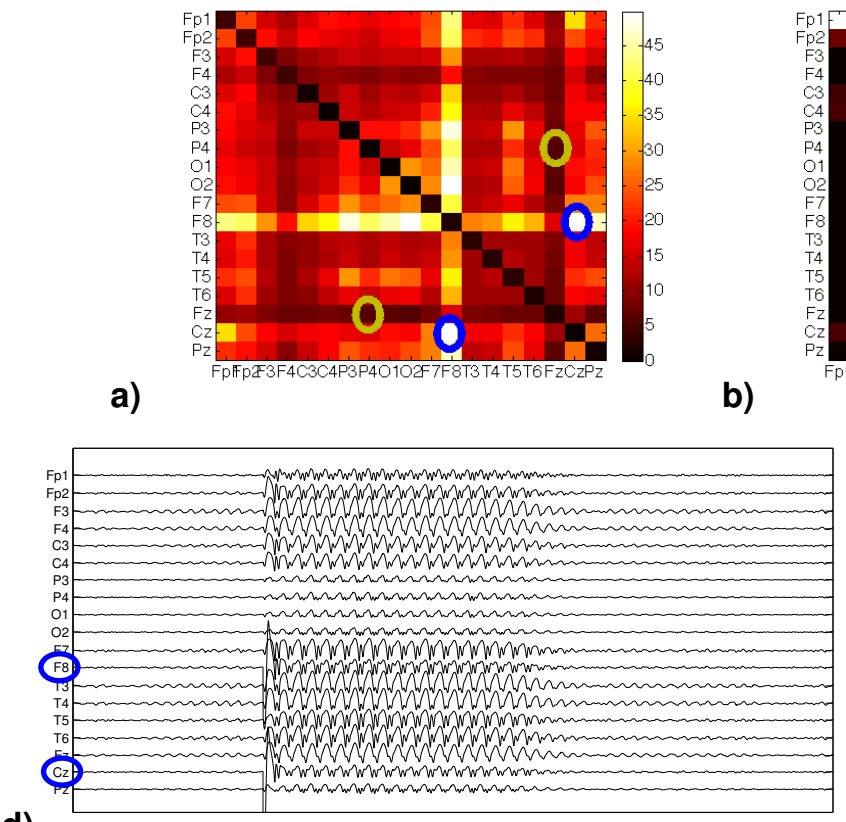

d)

b)

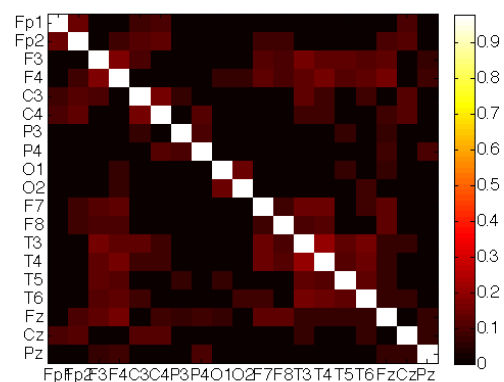

e)

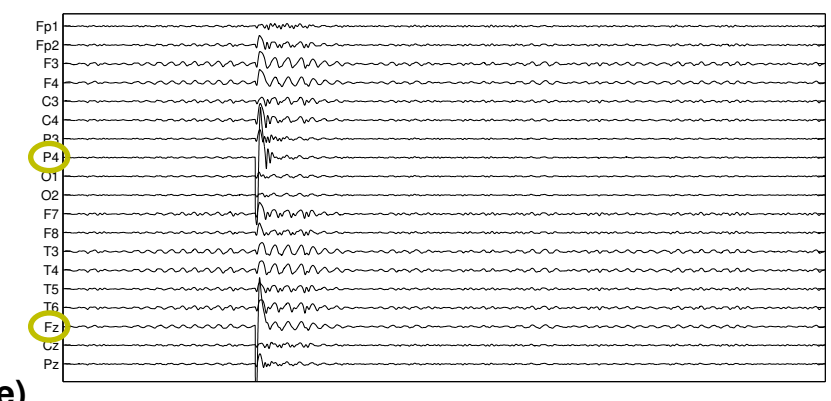

c)

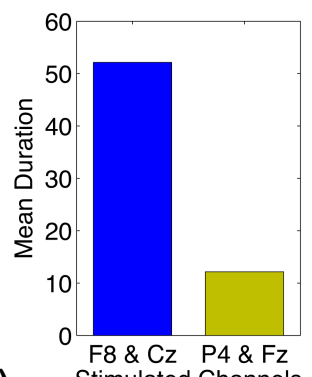

Stimulated Channels

Fig. 4. a) Color map of duration of response upon stimulation to channels as indicated on the $\mathrm{x}$ and $\mathrm{y}$ axes. Lighter colours indicate longer responses. b) Connectivity matrix of the model $(A)$. c) Mean seizure duration following stimulation to the selected channels in the stochastic system. d) Long seizure following stimulation of the F8 and Cz nodes in the stochastic system. e) Short seizure following stimulation of the F8 and Cz nodes in the stochastic system.

system. The underlying connectivity for which is shown in figure $4 b$.

A differential response, in terms of the resulting seizure duration, can be seen depending on which nodes are stimulated. Specifically, the stimulation of nodes $\mathrm{F} 8$ and $\mathrm{Cz}$ indicated by the blue circle results in a seizure with a long duration, an exemplary timeseries of which can be seen in figure $4 \mathrm{~d}$. Upon stimulation of nodes $\mathrm{P} 4$ and $\mathrm{Fz}$, indicated by the green circle, the resulting seizure is of a significantly shorter duration and of a lower amplitude, an example of which is shown in figure 4e. Stimuli to these two pairs of nodes in the model represent the maximal and minimal response respectively for a given stimulus strength of -3 . This is also the case in the stochastic system when considering the mean duration of the response over a repeated trials approach with different noise vectors. Plotted in figure $4 \mathrm{c}$ is the mean response duration for stimulation to each of the aforementioned node pairs. The deterministic system therefore serves as a good approximation to the stochastic system. Due to the stochasticity of the system the resulting seizure duration following stimulation can differ between trials. Initial exploration of the model in this regard suggests that the seizure duration follows an approximately exponential distribution with a mean as plotted in figure 5c. A study of seizure duration distributions in WAG/Rij epileptic rats indicated that spontaneous seizures also follow an exponential distribution (see figure 4 in [28]). Stimulations to a single node did not result in any seizure of significant duration. This is shown in the diagonals of figure $4 \mathrm{a}$.

To investigate the impact of different stimulus strengths $(s)$ the stimulus to the two pairs of nodes was scanned as a parameter. Figure 5 shows the resulting seizure duration for a given stimulation strength to either the $\mathrm{F} 8$ and $\mathrm{Cz}$ nodes

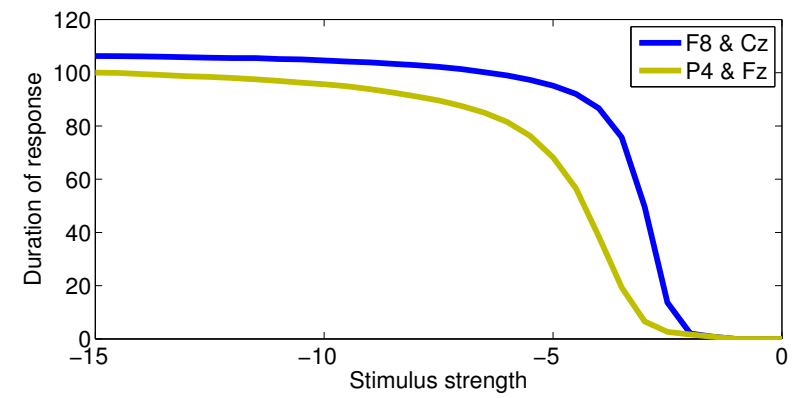

Fig. 5. Seizure duration as a result of a stimulus to selected nodes in the model. A larger stimulus is required to elicit a long seizure when stimulating th $\mathrm{P} 4 \& \mathrm{Fz}$ nodes as opposed to the F8 \& Cz nodes.

(blue line) or the $\mathrm{P} 4$ and $\mathrm{Fz}$ nodes (green line). For small stimulations $(-1.5 \lesssim s \lesssim 0)$ little to no response can be seen, i.e. a subthreshold response. For large stimuli the resulting duration saturates to an upper limit of around 110 timesteps. for mid-range stimuli (e.g. $-5 \lesssim s \lesssim-1.5$ ) the duration varies significantly, depending upon which regions are stimulated. The stimulation threshold of the P4 and Fz nodes is less steep and has a more graded response. This is in line with a recent experimental observation that a stimulus of a larger current is required in the primary somatosensory upper lip cortex (S1ULp) to induce a seizure than stimulation of the secondary somatosensory cortex (S2), see, e.g. figure 3d in [6].

In summary we have demonstrated that the model introduced by Taylor et al [24] can be expanded to account for multiple cortical regions and is capable of reproducing many key features observed in experimental and clinical epileptic seizures: 
1 Transitions between a low amplitude background and a high amplitude seizure state (cf. figures $2 b, 2 c$ and 3a, 3b).

2 Transitions between irregular background oscillations and repeating SWD oscillations (cf. figures $2 \mathrm{~b}$, $2 \mathrm{c}$ and $3 \mathrm{a}, 3 \mathrm{~b}$ ).

3 The morphology of the SWD waveform during the seizure (cf. $3 a$ and $3 b$ )

4 Transitions from a higher frequency background state to a lower frequency seizure state with higher harmonics (cf. lower panels in figures $2 a$ and $2 b$ ). Stimulus induced SWD (cf. figure 2a and figure 3 in ref [5])

6 Termination of SWD without the need for a further stimulus (cf. figure 2a and figure 3 in ref [5]).

$7 \quad$ Large amplitude SWD across multiple channels (cf. figures $3 a$ and $3 b$ ).

8 Amplitude and waveform variation between different channels in space (cf. figures $3 a$ and $3 b$ ).

9 Synchrony between channels during the seizure (cf. figures $3 a$ and $3 b$ ).

10 A larger stimulus is required to elicit a seizure dependant upon the location of the stimulation (cf. figures 4,5 and figure $3 \mathrm{~d}$ in ref [6])

11 Upon stimulation of the same intensity, different responses can be seen depending on the location of the stimulus (cf. figure 4 and table 1 in [5] and figure $3 d$ in [6]).

\section{CONCLUSION}

In this study we have presented a model of spatially extended thalamocortical interaction to study epileptic spike and wave discharges and the impact of stimulation. We showed that the model can reproduce many of the clinically observed phenomena in the time, space and frequency domains. Furthermore we have shown that a seizure can be simulated as a transient response to a stimulation. Finally, in agreement with recent experimental findings, the duration of the seizure crucially depends on the spatial location of the stimulus and its amplitude.

The notion of epilepsy as a low dimensional dynamical disease was pioneered by Milton [29] and Lopes da Silva [30] amongst others. Specifically, in [31] Lopes da Silva demonstrated that the addition of a noise term to a bistable system can lead to transitions between background and seizure-like states. Since that system is bistable the mechanism for offset is the same as that for onset. In the model presented in this work there is a key difference, seizures are a transient response to a stimulus, there is only one stable state used - the background state. This means that seizures can terminate autonomously without the need for further input or noise. To our knowledge this mechanism has not been reported before in a spatially extended thalamocortical model of SWD. Other mechanisms of onset and offset include intermittency [17] and through the use of a parameter ramp [15].

Until recently [17], [20], [22] previous models of SWD at the macroscopic scale have focused on the temporal aspects of SWD [14], [15], [32]. However, it has been suggested that investigation of the heterogeneous spatial aspects of SWD could lead to an improved understanding and more effective treatments [3]. Further evidence for the importance of spatial variation comes from the patterns in activated regions of the cortex during fMRI recordings of absence seizures [11]. These were found to be heterogeneous and patient specific. It is therefore crucial that models incorporate both spatial and temporal aspects [4].

Through the incorporation of heterogeneous connectivity realistic epileptic waveform morphologies were observed without the need for exhaustive parameter fitting. A previous work to fit model output to EEG in a quantitative approach used a genetic algorithm to alter three model parameters over time [23]. In that work it was suggested that, following the modulation of the parameters, patients could be grouped according to their clinical diagnosis and treatment outcome. The capability of the present model to produce realistic waveforms means that such a study could be performed here, crucially however, spatial information could be included in addition to the temporal data used in [23]. The similarity between our model output and clinical EEG recordings is not to be understated here. This could be crucial for the development of patient specific models where it is necessary to reproduce specific aspects of the data. Our model can represent an important first step towards achieving this goal.

In this contribution we have used our model to investigate the impact of stimulation during a background state to initiate a seizure. We have found that the resulting dynamics depend on the spatial location of the stimulus. This is in agreement with recent experimental findings in both the WAG/Rij and GAERS strains of epileptic rats [5], [6]. In our model this depends on the underlying structural connectivity. As a first approximation of structural connectivity we used the correlation between the channels to infer connectivity in our model. The underlying connectivity will differ depending on the measure used and the patient to be investigated. Many different approaches can be used based on either inferred functional connectivity (e.g. correlation, mutual information, phase coherence etc) or structural connectivity (e.g. tract tracing, diffusion tensor imaging, diffusion spectrum imaging etc). Determining which measure most appropriately describes the anatomical network is an open issue in neuroscience and is left as future work. It is noteworthy that the relationship between stimulus response and connectivity is nontrivial (cf. figure $4 \mathrm{a}$ and figure $4 \mathrm{~b}$ ). However, recent research into elucidating connections through stimulation [33] could help provide further insights in this direction in the near future.

One implication of our modelling results here is that we find some cortical regions are more likely to induce a seizure when stimulated than others when given a stimulus of the same amplitude. Since the connections between the thalamus and the cortex in the model are homogeneous this means that cortico-cortico transmission plays a critical role in our simulations of seizure initiation. Furthermore, the involvement of the thalamus is also crucial for seizure maintenance. The model therefore makes the specific model based prediction that heterogeneities in cortico-cortical connectivity can serve 
as one possible explanation for the existence of the cortical focus. An alternative hypothesis could be that the focus is simply intrinsicly more excitable which, in the context of modelling, would infer it has a different parameter value. In future work the model could be used to test specific network based hypotheses to help guide experimentalists. For example, the impact of a lesion can be explicitly simulated. Through the use of in silico experimentation many more lesions can be performed to identify the optimal location to lesion in order to reduce seizures. This can then be tested experimentally and the results used to better inform the model. Furthermore, inspiration from control theory [34] and synchronisation theory [35], [36] could be drawn to inform predictions in such a framework.

Ultimately, it is crucial that both mathematical and experimental models are developed in conjunction in order to improve our understanding of epilepsy as a spatio-temporal disease.

\section{ACKNOWLEDGMENT}

We thank H. Muhle, M. Siniatchkin, F. Moeller and U. Stephani, Neurology, University Hospital Kiel and K. Schindler, Department of Neurology, Inselspital, Bern, CH for clinical EEG data and discussion of neurological matters.

\section{REFERENCES}

[1] D. Bosnyakova, A. Gabova, A. Zharikova, V. Gnezditski, G. Kuznetsova and G. Van Luijtelaar, "Some peculiarities of time-frequency dynamics of spike-wave discharges in humans and rats," Clinical neurophysiology, vol. 118, no. 8, pp. 1736-1743, 2007.

[2] E. Rodin and O. Ancheta, "Cerebral electrical fields during petit mal absences," Electroencephalography and Clinical Neurophysiology, vol. 66, no. 6 , pp. 457-466, 1987.

[3] H. Blumenfeld, "Cellular and network mechanisms of spike-wave seizures," Epilepsia, vol. 46, pp. 21-33, 2005.

[4] G. Baier, M. Goodfellow, P. Taylor, Y. Wang, and D. Garry, "The importance of modelling epileptic seizure dynamics as spatio-temporal patterns," Frontiers in Physiology, vol. 3, p. 281, 2012.

[5] A. Lüttjohann, S. Zhang, R. de Peijper, and G. van Luijtelaar, "Electrical stimulation of the epileptic focus in absence epileptic wag/rij rats: assessment of local and network excitability," Neuroscience, vol. 188, pp. 125-134, 2011.

[6] T. Zheng, T. OBrien, M. Morris, C. Reid, V. Jovanovska, P. OBrien, L. van Raay, A. Gandrathi, and D. Pinault, "Rhythmic neuronal activity in s2 somatosensory and insular cortices contribute to the initiation of absence-related spike-and-wave discharges," Epilepsia, 2012.

[7] D. Pinault and T. O'Brien, "Cellular and network mechanisms of genetically-determined absence seizures," Thalamus \& Related Systems, vol. 3 , no. 3 , p. 181,2005

[8] H. Meeren, J. Pijn, E. Van Luijtelaar, A. Coenen, and F. Lopes da Silva, "Cortical focus drives widespread corticothalamic networks during spontaneous absence seizures in rats," The Journal of Neuroscience, vol. 22, no. 4, p. 1480, 2002.

[9] H. Meeren, G. van Luijtelaar, F. Lopes da Silva, and A. Coenen, "Evolving concepts on the pathophysiology of absence seizures: the cortical focus theory," Archives of neurology, vol. 62, no. 3, p. 371, 2005.

[10] H. Nersesyan, F. Hyder, D. Rothman, and H. Blumenfeld, "Dynamic fmri and eeg recordings during spike-wave seizures and generalized tonic-clonic seizures in wag/rij rats." Journal of Cerebral Blood Flow and Metabolism, vol. 24, no. 6, p. 589, 2004.

[11] F. Moeller, P. LeVan, H. Muhle, U. Stephani, F. Dubeau, M. Siniatchkin, and J. Gotman, "Absence seizures: Individual patterns revealed by eegfmri," Epilepsia, vol. 51, no. 10, pp. 2000-2010, 2010.

[12] M. Steriade and D. Contreras, "Spike-wave complexes and fast components of cortically generated seizures. i. role of neocortex and thalamus," Journal of neurophysiology, vol. 80, no. 3, pp. 1439-1455, 1998.
[13] A. Destexhe, "Spike-and-wave oscillations based on the properties of GABAB receptors," The Journal of Neuroscience, vol. 18, no. 21, p. 9099, 1998

[14] P. A. Robinson, C. J. Rennie, and D. L. Rowe, "Dynamics of large-scale brain activity in normal arousal states and epileptic seizures," Physical Review E, vol. 65, no. 4, p. 041924, Apr 2002.

[15] M. Breakspear, J. Roberts, J. Terry, S. Rodrigues, N. Mahant, and P. Robinson, "A unifying explanation of primary generalized seizures through nonlinear brain modeling and bifurcation analysis," Cerebral Cortex, vol. 16, no. 9, p. 1296, 2006.

[16] F. Marten, S. Rodrigues, P. Suffczynski, M. Richardson, and J. Terry, "Derivation and analysis of an ordinary differential equation mean-field model for studying clinically recorded epilepsy dynamics," Physical Review E, vol. 79, no. 2, p. 021911, 2009.

[17] M. Goodfellow, K. Schindler, and G. Baier, "Intermittent spike-wave dynamics in a heterogeneous, spatially extended neural mass model," NeuroImage, vol. 55, no. 3, pp. 920-932, 2011.

[18] H. Wilson and J. Cowan, "Excitatory and inhibitory interactions in localized populations of model neurons," Biophysical Journal, vol. 12, no. 1, pp. 1-24, 1972.

[19] S. Amari, "Dynamics of pattern formation in lateral-inhibition type neural fields," Biological Cybernetics, vol. 27, no. 2, pp. 77-87, 1977.

[20] P. Taylor and G. Baier, "A spatially extended model for macroscopic spike-wave discharges," Journal of Computational Neuroscience, vol. 31, no. 3, pp. 679-684, 2011 .

[21] Y. Wang, M. Goodfellow, P. Taylor, and G. Baier, "Phase space approach for modeling of epileptic dynamics," Physical Review E, vol. 85, no. 6, p. 061918, 2012.

[22] P. Taylor, M. Goodfellow, Y. Wang, and G. Baier, "Towards a large-scale model of patient-specific epileptic spike-wave discharges," Biological Cybernetics, 2012.

[23] A. Holgado-Nevado, F. Marten, M. Richardson, and J. Terry, "Characterising the dynamics of eeg waveforms as the path through parameter space of a neural mass model: Application to epilepsy seizure evolution," NeuroImage, vol. 59, p. 2374, 2012.

[24] P. Taylor, Y. Wang, M. Goodfellow, F. Moeller, U. Stephani, and G. Baier, "Stimulus driven spike-wave seizure abatement," 2013 (submitted).

[25] H. A. Swadlow and S. G. Waxman, "Axonal conduction delays," vol. 7, no. 6 , p. 1451, 2012.

[26] A. Aarabi, F. Wallois, and R. Grebe, "Does spatiotemporal synchronization of EEG change prior to absence seizures?" Brain Research, vol. 1188 , pp. 207-221, 2008.

[27] G. Parker, H. Haroon, and C. Wheeler-Kingshott, "A framework for a streamline-based probabilistic index of connectivity (pico) using a structural interpretation of mri diffusion measurements," Journal of Magnetic Resonance Imaging, vol. 18, no. 2, pp. 242-254, 2003.

[28] P. Suffczynski, S. Kalitzin, and F. Lopes Da Silva, "Dynamics of non-convulsive epileptic phenomena modeled by a bistable neuronal network," Neuroscience, vol. 126, no. 2, pp. 467-484, 2004.

[29] J. Milton, "Epilepsy: multistability in a dynamic disease," Self-organized biological dynamics \& nonlinear control: toward understanding complexity, chaos, and emergent function in living systems, p. 374, 2000.

[30] F. Lopes Da Silva, W. Blanes, S. Kalitzin, J. Parra, P. Suffczynski, and D. Velis, "Epilepsies as dynamical diseases of brain systems: basic models of the transition between normal and epileptic activity,' Epilepsia, vol. 44, pp. 72-83, 2003.

[31] F. da Silva, W. Blanes, S. Kalitzin, J. Parra, P. Suffczynski, and D. Velis, "Dynamical diseases of brain systems: different routes to epileptic seizures," Biomedical Engineering, IEEE Transactions on, vol. 50, no. 5, pp. 540-548, 2003.

[32] F. Marten, S. Rodrigues, O. Benjamin, M. Richardson, and J. Terry, "Onset of polyspike complexes in a mean-field model of human electroencephalography and its application to absence epilepsy," Philosophical Transactions of the Royal Society A: Mathematical, Physical and Engineering Sciences, vol. 367, no. 1891, p. 1145, 2009.

[33] R. Matsumoto, D. Nair, E. LaPresto, W. Bingaman, H. Shibasaki, and H. Lüders, "Functional connectivity in human cortical motor system: a cortico-cortical evoked potential study," Brain, vol. 130, no. 1, pp. 181-197, 2007

[34] Y. Liu, J. Slotine, and A. Barabási, "Controllability of complex networks," Nature, vol. 473, no. 7346, pp. 167-173, 2011.

[35] Q. Pham and J. Slotine, "Stable concurrent synchronization in dynamic system networks," Neural Networks, vol. 20, no. 1, pp. 62-77, 2007.

[36] W. Wang and J. Slotine, "On partial contraction analysis for coupled nonlinear oscillators," Biological cybernetics, vol. 92, no. 1, pp. 38-53, 2005 . 